\title{
Gram Negative Cocci Measurement
}

National Cancer Institute

\section{Source}

National Cancer Institute. Gram Negative Cocci Measurement. NCI Thesaurus. Code C161401.

The determination of the amount of gram negative cocci present in a sample. 